Voix et Images

voixetimages

\title{
Entretien avec Louise Dupré
}

\section{Janet M. Paterson}

Volume 34, numéro 2 (101), hiver 2009

Louise Dupré

URI : https://id.erudit.org/iderudit/029462ar

DOI : https://doi.org/10.7202/029462ar

Aller au sommaire du numéro

Éditeur(s)

Université du Québec à Montréal

ISSN

0318-9201 (imprimé)

1705-933X (numérique)

Découvrir la revue

Citer ce document

Paterson, J. M. (2009). Entretien avec Louise Dupré. Voix et Images, 34(2), 11-23. https://doi.org/10.7202/029462ar d'utilisation que vous pouvez consulter en ligne.

https://apropos.erudit.org/fr/usagers/politique-dutilisation/ 


\title{
ENTRETIEN AVEC LOUISE DUPRÉ
}

\author{
JANET M. PATERSON \\ Université de Toronto
}

voIX ET IMAGES Pourriez-vous, pour commencer, retracer votre parcours de votre ville natale à Montréal, de votre enfance à votre venue à l'écriture?

LOUISE DUPRÉ Je suis née à Sherbrooke, en Estrie. Je suis l'aînée d'une famille modeste : mon père était ouvrier. Ma mère cependant vient d'une famille cultivée et très avant-gardiste: sa grand-mère était pianiste, son père est allé étudier le métier de tailleur à New York, il lisait des livres à l'Index, il était athée, ce qui était un scandale à l'époque. Mon goût de la littérature me vient donc de ma famille maternelle. J'ai fait un baccalauréat et une maîtrise en littérature à l'Université de Sherbrooke. J'ai vécu ensuite à Thetford Mines, où j'ai enseigné au cégep. Thetford Mines, on le sait, est une ville minière qui a été marquée par une grève historique en 1949. Dans les années 1970, les Thetfordois avaient encore une conscience politique et sociale très aiguisée. J'ai commencé à m'impliquer socialement: je suis devenue militante syndicale. Mais je me suis rendu compte que je ne pouvais pas à la fois enseigner, militer et écrire régulièrement. J'ai laissé mon poste syndical pour me tourner vers l'écriture.

VoIX ET IMAGES Quand, au juste, avez-vous commencé à écrire de la poésie et de la fiction? Y a-t-il des circonstances particulières qui vous ont menée vers un genre ou l'autre?

LOUISE DUPRÉ Lorsque j'étais à l'école primaire, j'écrivais de petites pièces de théâtre qu'on montait après la classe. À l'adolescence, je me suis mise à faire des poèmes, sur des amours impossibles, comme beaucoup d'adolescents. Mais j'ai vraiment commencé à écrire à Thetford Mines. Au cégep, nous écrivions des chansons, des pièces de théâtre parodiques que nous montions ensuite pour les étudiants. Puis le comité de condition féminine de la ville nous a demandé, à moi et à d'autres femmes, dont la poète Louise Cotnoir, d'écrire et de monter un spectacle pour le 8 mars 1975. Ainsi est né Si Cendrillon pouvait mourir!, qui a été ensuite publié par les Éditions du remue-ménage. C'est à cette époque-là que j'ai voulu m'engager vraiment dans l'écriture.

Voix ET IMAGES Et vous avez publié vos premiers textes de poésie...

LOUISE DUPRÉ Oui, à l'époque, j'ai découvert l'écriture des femmes, alors en pleine effervescence, qui abordait des thématiques nouvelles, audacieuses, de même 
qu'elle s'inscrivait dans des formes hybrides, postmodernes - même si on disait modernes à l'époque, puisque le mot postmoderne n'était pas encore connu au Québec. J'étais abonnée aux Herbes rouges et à La Nouvelle Barre du jour, et je lisais tout ce qu'on y faisait paraître. J'ai d'ailleurs publié mon premier texte à La Nouvelle Barre du jour en 1978, dans un numéro dirigé par Nicole Brossard. J'avais mis un texte à la poste et j'avais attendu la réponse. Vous ne pouvez pas savoir le bonheur que j'ai ressenti quand j'ai appris que mon texte avait été retenu.

VoIX ET IMAGES La fiction est venue plus tard?

LOUISE DUPRÉ J'ai commencé à écrire des nouvelles parce que des amis préparaient des numéros de revues, puis je me suis mise à mon premier roman bien après, même si je rêvais d'écrire un roman depuis longtemps. J'enseignais, j'avais un jeune enfant, de sorte que les genres brefs convenaient mieux à ma vie fragmentée. On parle peu du rapport entre la pratique de certains genres littéraires et les conditions d'écriture que connaissent les écrivains. Mais le lien est clair. J'ai pu mener à terme La memoria pendant un congé sabbatique. J'avais commencé le livre auparavant, bien sûr, mais il me fallait une longue période de liberté pour en faire une dernière version qui me paraîtrait satisfaisante.

voIX ET IMAGES L'écriture de la prose a-t-elle eu des résonances sur votre travail poétique?

LOUISE DUPRÉ Quand j'ai écrit Noir déjā, j'avais déjà publié des nouvelles et j'avais commencé mon roman La memoria. Jusque-là, mes recueils de poésie étaient composés de suites poétiques en prose, une prose narrative, hybride, qui présentait des personnages, un contexte, les bribes d'une histoire, etc. Le désir de raconter et le désir du lyrisme étaient étroitement imbriqués. Mais l'écriture romanesque a fait en sorte que j'ai séparé les deux. Non pas complètement: actuellement, les frontières entre les genres littéraires sont mouvantes, pour ne pas dire floues ou carrément imprécises, selon les auteurs, ce qui est très bien. Mais avec Noir déjà, j'ai éprouvé le désir de travailler le vers, que je n'avais pas beaucoup travaillé jusque-là. Il y a dans le vers une rigueur qui me rejoint, qui rejoint, en fait, le plaisir de sculpter la matière linguistique comme on sculpterait le marbre.

voIX ET IMAGES Vous êtes cependant revenue à la prose avec votre recueil Tout près...

LOUISE DUPRÉ En effet. Mais les suites poétiques de Tout près sont moins narratives que celles de La peau familière, de Chambres et de Bonheur, du moins il me semble. Ici, la poésie a moins souci de raconter que de présenter une réflexion, ce qui implique une posture plus philosophique. Serait-il juste de dire que ce recueil est plus près de l'essai que de la narrativité ? Peut-être. Mais les suites poétiques de Tout près sont séparées de poèmes en vers. Je n'ai pas pu renoncer complètement au vers.

voIX ET IMAGES $\bar{A}$ la différence de nombreux professeurs d'université, vous avez choisi cette profession après plusieurs années d'enseignement au cégep. Pouvezvous déterminer le tournant qui vous a menée au doctorat?

LOUISE DUPRÉ En effet, je n'ai pas eu une "carrière» en ligne droite. Et si j'ai bénéficié de bourses d'excellence pour la maîtrise et le doctorat, je ne pourrais sans doute plus en bénéficier aujourd'hui: les étudiants doivent montrer un cursus parfait depuis le collégial. En 1981, j'ai entrepris un doctorat par passion pour l'écriture. Je 
faisais déjà des recherches sur l'écriture au féminin, je participais à des colloques sur le sujet, je m'intéressais vivement à la nouvelle poésie, j'écrivais moi-même: j'ai voulu me donner du temps pour lire, réfléchir et approfondir mes recherches.

voix ET IMAGES Pour votre thèse de doctorat, vous avez étudié l'œuvre de Nicole Brossard, Madeleine Gagnon et France Théoret dans une perspective féministe. Êtesvous consciente d'inscrire une subjectivité féminine dans votre poésie et vos romans?

LOUISE DUPRÉ Je suis convaincue que les chercheurs passionnés s'intéressent à un sujet particulier par nécessité personnelle. Ce que la perspective-femme m'a permis de découvrir, c'est un autre point de vue sur la littérature, une autre réalité du sujet, correspondant à ce que je ressentais depuis longtemps sans pouvoir le nommer: l'impression d'être à côté, de travers, dans la marge. D'être une autre, en somme, de me sentir aliénée... Je ne reconnaissais pas ma réalité dans les œuvres que je lisais. Par exemple, je suis une grande admiratrice de Flaubert et, quand j'étais étudiante au baccalauréat, je me souviens avoir défendu Madame Bovary : j'avais l'impression qu'on ne comprenait pas vraiment ce personnage. Grâce aux études féministes, à la psychanalyse et aux artistes femmes, on connaît mieux aujourd'hui les mécanismes de la psyché féminine, mais il reste encore tant à apprendre. Et c'est à cela que je veux travailler, c'est la petite pierre que je voudrais ajouter à la recherche.

voIX ET IMAGES Vous avez mentionné que, pendant les années 1970, vous étiez militante syndicale à Thetford Mines. Ce militantisme a-t-il influencé votre perspective féministe ou bien vous situez-vous plus près d'une écriture engagée?

LOUISE DUPRÉ Je me sens près de la conception de Simone de Beauvoir à qui on reprochait de n'être pas militante dans ses textes. Certaines écrivaines ont écrit de beaux textes avec une ferveur militante dans les années 1970. Mais l'écriture doit se renouveler: c'est le défi que se donne chaque génération d'écrivains. J'ai commencé à écrire dans les années 1980 : j'appartiens à une autre génération, celle de l'intime. Mais qu'est-ce que l'intime? Cette appellation a prêté à beaucoup de lieux communs, comme l'affirmation que les écrivains de l'intime sont tournés vers leurs blessures, qu'ils sont narcissiques, qu'ils ne sont pas engagés. Or, travailler sur la subjectivité féminine, sur ses désirs inconscients, ses douleurs, est pour moi un motif d'engagement profond. Sans doute à cause de mes études me revient cette phrase de Socrate : «Connais-toi toi-même.» L'être humain ne peut avancer s'il reste aveugle à ce qu'il est. Il répète le passé, il n'y a pas de possibilité de renouvellement.

Voix ET IMAGES Vous évoquez la douleur et la blessure. Or, ces thèmes sont effectivement présents dans votre œuvre, autant dans La Voie lactée que dans votre dernier recueil de nouvelles, L'été funambule. Pratiquez-vous ce qu'on pourrait appeler une «écriture de la blessure»? Que représente pour vous ce genre d'écriture?

LOUISE DUPRÉ Votre question me touche, et cela d'autant plus qu'on m'a demandé, en 2003, de présenter l'exposé inaugural de la 33e Rencontre québécoise internationale des écrivains sur le thème de "L'écrivain/e et la blessure ». J'aime d'ailleurs beaucoup votre expression, «une écriture de la blessure». Je travaille effectivement à partir de la blessure et de la douleur qui s'y rattache, puisque je travaille sur la subjectivité, les traces du passé, les marques laissées par la petite histoire, évidemment en lien avec la grande, pour justement déterrer ce qui est caché, le mettre en 
lumière, mieux le comprendre. J'ai toujours essayé de faire en sorte que mon écriture échappe au refoulement, sans jamais y parvenir complètement, évidemment...

Certaines questions se posent cependant aujourd'hui : comment écrire la blessure dans un monde de «reality show» où l'individu adore s'exhiber pour avoir ses quinze minutes de gloire? Comment faire en sorte que la blessure ne se donne pas en spectacle, que l'écriture de la blessure ne devienne pas sensationnaliste? Je crois que c'est en gardant une pudeur, en essayant de travailler à une voix qui peut parfois pleurer, parfois crier, parfois haleter, parfois montrer sa colère, mais refuse le tapeà-l'œil. Une voix qui sait malgré tout garder une distance avec les faits... C'est ce que permet justement le travail de l'écriture.

voIX ET IMAGES Quels poètes, romanciers, romancières vous ont le plus marquée? Le plus influencée?

LOUISE DUPRÉ Le premier travail de recherche que j'avais choisi de faire, quand j'étais au collège, portait sur Les pensées de Pascal; le deuxième, sur Les fleurs du mal, de Baudelaire. J'ai beaucoup lu le thêâtre du xvire siècle, Racine en particulier, que j'adorais. Puis les romantiques. À l'époque, nous recevions un enseignement très axé sur la littérature française; j'étudiais d'ailleurs dans un collège français. Dans nos cours d'anglais, nous lisions des poètes anglais: je me souviens de cours sur Keats, Shelley, etc. Je ne peux vraiment mesurer les conséquences de ces lectures sur mon écriture, mais je remarque que, autant dans ma prose que dans ma poésie, la scansion est très importante, de même que les sonorités.

J'ai connu la littérature québécoise à l'université, avec Joseph Bonenfant et Antoine Sirois. J'ai découvert Laure Conan, Albert Laberge, Gabrielle Roy, Anne Hébert. Et des poètes comme Saint-Denys Garneau, puis l'Hexagone. J'ai d'ailleurs fait ma maîtrise sur Fernand Ouellette. La lecture de Dans le sombre avait été pour moi une révélation, pour l'alliance entre la sexualité très prégnante et la spiritualité. Puis, comme je l'ai précisé, j'ai découvert les poètes de la modernité québécoise. C'est dans Voix et Images que j'ai publié mon premier compte rendu: il portait sur Veiller ne plus veiller de Michel van Schendel.

voix ET IMAGES Comment vous situez-vous parmi les écrivains de la dernière décennie?

LOUISE DUPRÉ Mes premiers textes ont été publiés dans La Nouvelle Barre du jour. C'était une excellente école et je garde un lien très fort avec les poètes de «la nouvelle écriture», qui continuent d'ailleurs leur parcours. Plusieurs sont passés à la nouvelle, au récit, au roman. Mais tout écrivain éprouve aussi la nécessité d'assumer son propre imaginaire, sa voix propre, ce qui suppose un espace de liberté. Dans La peau familière, en 1983, j'avais cité Roland Barthes, qui affirmait: "Il m'est devenu indifférent de ne pas être moderne», ce qui avait choqué certains poètes. Tant pis : il faut accepter de décevoir, de choquer... Ce qui caractérise peut-être mon écriture, c'est une tension constante et irrémédiable entre l'inquiétude, voire le tourment, et la sérénité, comme entre la force et la fragilité.

voIX ET IMAGES Malgré une variété d'influences littéraires, vous avez consacré toutes vos recherches à la littérature québécoise. Était-ce un choix conscient et motivée

LOUISE DUPRÉ Si je me suis consacrée à la littérature québécoise, c'est qu'il y avait relativement peu de travaux sur notre littérature à l'époque, tout particulièrement 
sur la poésie : je trouvais qu'il fallait s'y pencher. C'est un engagement que je me suis donné. Car comment développer une culture québécoise riche si on ne connaît pas - et ne reconnaît pas - sa littérature? Cette question revient actuellement avec beaucoup d'acuité dans les débats sur l'enseignement de la littérature au collégial.

VOIX ET IMAGES Est-ce aussi une question importante pour l'enseignement universitaire et pour la direction des thèses et des mémoires?

LOUISE DUPRÉ Effectivement, l'enseignement de la littérature québécoise est primordial à l'université, surtout à une époque où la littérature occupe de moins en moins d'espace symbolique au Québec, à une époque où le livre appartient à «l'industrie culturelle», où on a l'impression que l'importance d'un écrivain est relative à sa visibilité. Nous pourrions en parler longuement...

VoIX ET IMAGES Plusieurs critiques ont remarqué la présence du quotidien, du détail dans votre œuvre. Pourquoi portez-vous tant d'attention aux détails, à ce qui pourrait paraître insignifiant, comme par exemple la tasse de thé dans Tout comme elle? LOUISE DUPRÉ Je me donne la tâche de faire signifier l'in-signifiant. De faire voir ce qu'on ne voit pas. De faire exister ce qui n'existe pas. On est actuellement dans une culture du sensationnalisme, qui insiste sur ce qui crève les yeux, en remet. Mon regard, même au théâtre ou dans le roman, reste un regard de poète: les poètes prêtent attention au petit, au banal pour en faire ressortir la beauté ou l'exactitude. Je rejoins sans doute aussi les artistes visuels et ceux de la vidéo, qui souvent prêtent attention au minuscule, au minimal.

Mais souvent dans un texte, un seul objet recrée tout un monde, la tasse de thé dans Tout comme elle, par exemple. La mère ne boit ni du vin ni du café, mais du thé. Ce sont sans doute mes origines sherbrookoises qui reviennent. Sherbrooke, à l'origine, était une ville loyaliste. Et certaines habitudes anglophones sont restées dans les coutumes, même si la ville est devenue francophone avec le temps.

voIX ET IMAGES Le recueil de poèmes Les mots secrets est destiné à des adolescents de 11 à 14 ans. Qu'est-ce qui vous a poussée à écrire pour un jeune public? Était-ce une façon de renouer avec votre enfance?

LOUISE DUPRÉ Les éditions de La courte échelle ont décidé de mettre sur pied une collection de poésie pour les adolescents et Sylvie Massicotte, la directrice de la collection, m'a invitée à écrire un texte. D'abord, j'ai décliné l'invitation, puis les mots me sont venus, doucement. J'ai compris que je n'avais pas à écrire pour les adolescents, mais à partir de la petite fille que j'avais été. Une enfant qui n'était bien ni avec elle-même ni avec les autres, comme beaucoup d'enfants à la puberté, et qui avait trouvé, dans la lecture et l'écriture, matière à mieux se comprendre et à mieux comprendre le monde. Matière à vivre aussi. À la relecture, je me suis rendu compte que les sentiments, les sensations que j'abordais n'étaient pas uniquement vécus par des fillettes, mais aussi par des garçons. Alors, j'ai enlevé toutes les marques de sexuation, de sorte que le je peut être aussi bien celui d'un garçon que d'une fille. Ce qui m'a fait plaisir d'ailleurs, c'est qu'un jeune garçon m'a écrit pour me dire qu'il se retrouvait dans le texte.

Voix ET IMAGES Enlever les marques de la sexuation dans l'écriture n'est pas évidemment toujours possible et surtout pour une critique et une écrivaine féministe! Quelles sont les limites de la sexuation dans l'écriture? 
LOUISE DUPRÉ Justement, l'arrivée d'une troisième vague du féminisme a fait comprendre de façon moins dichotomique la question de la différence sexuelle. Chaque individu a intégré à sa façon les traits de la sexuation. Et les a défiés aussi. L'écriture est précisément le lieu de l'individuel, le lieu où l'on peut remettre en question les modèles qui ont été les nôtres. C'est aussi le lieu où se dévoile une altérité et les fantasmes qui y sont liés. Dans La Voie lactée, par exemple, le personnage d'Alessandro m'apparaît comme un personnage maternel : je ne m'en suis pas aperçue au moment où j'écrivais le roman mais, a posteriori, il me semble que ce n'est pas un hasard si, au moment où Anne décide de mettre un océan entre elle et sa mère, elle se retrouve avec un homme qui a l'âge de sa mère, un homme qui en tient lieu, un homme enveloppant, compréhensif...

VoIX ET IMAGES Prenons maintenant la question par un autre biais. Dans quelle mesure les marques de la sexuation peuvent-elles transformer l'écriture en y inscrivant un sujet féminin? Est-ce un facteur important pour vous?

LOUISE DUPRÉ En fait, c'est la subjectivité qui m'importe, mais une subjectivité qui cherche à saisir son rapport à la réalité, et qui par conséquent interroge le lien à la sexuation sans se réduire à des conception admises. Car le sujet féminin - comme tout sujet - est multiple, changeant, migrant. C'est en cela qu'il m'intéresse. Qu'estce que la féminité aujourd'hui ? On ne pourrait sans doute plus la définir comme au début du $\mathrm{xx}^{\mathrm{e}}$ siècle, même si les rêves des jeunes femmes ne diffèrent pas totalement, me semble-t-il, de ceux de leurs grands-mères. L'art est précisément le lieu où l'on peut aborder certaines questions sans avoir à y répondre de façon globale, sociologique.

VoIX ET IMAGES Vous avez écrit Tout comme elle en sachant que ce texte donnerait lieu à une représentation théâtrale.

LOUISE DUPRÉ Tout comme elle vient d'un projet avec Brigitte Haentjens, la metteure en scène bien connue. Nous avons décidé ensemble de la thématique. En effet, la relation mère-fille nous intéressait fortement toutes deux: il s'agit du nœud de la subjectivité féminine. Je connaissais le travail de Brigitte, qui aime recréer les textes des auteurs qu'elle monte, ce qu'elle a fait par exemple avec le roman Malina d'Ingeborg Bachmann. Je savais que je n'avais pas à écrire une pièce de thêâtre avec des répliques. Nous nous sommes laissé à toutes deux une grande liberté, moi pour le texte, Brigitte pour l'interprétation du texte. Le texte et le spectacle sont différents. Et c'est très bien ainsi.

voIX ET IMAGES Cette pièce, comme le texte qui l'inspire, est traversée par le thème de la filiation maternelle. Elle met au jour, à certains moments, une image idéalisée de la mère (belle et affectueuse) et idéalisée aussi de la relation mère-fille. D'autres scènes, toutefois, représentent de manière frappante l'incommunicabilité entre les générations de femmes. La filiation maternelle est-elle au centre de la pièce et de votre œuvre ainsi que l'affirment certains critiques?

LOUISE DUPRÉ Le rapport entre mère et fille est au centre de la psyché féminine. Il s'agit de la relation la plus complexe que vit une femme, à mon avis, une relation d'une grande richesse, mais aussi d'une grande violence puisque la fille devra se différencier de sa semblable et que la mère devra permettre à sa fille de se séparer d'elle. Et dans Tout comme elle, j'ai voulu montrer ce mouvement en ses différentes 
phases: fascination, agressivité, distanciation, incommunicabilité, réconciliation, etc. Certes, j'avais abordé cette relation dès mon premier recueil de poésie, La peau familière. Elle revient dans le recueil Tout près, mais aussi et surtout dans ma prose: dans mes romans et dans plusieurs nouvelles de L'été funambule. Il se pourrait bien que ce soit le noyau de ma démarche.

voIX ET IMAGES Quel effet la pièce a-t-elle eu sur vous? Sur votre façon de concevoir votre écriture?

LOUISE DUPRÉ Il est très stimulant de travailler avec un artiste d'une autre discipline, parce qu'on est amené à sortir de ses repères habituels, à se renouveler. Brigitte Haentjens a une façon très particulière de travailler: la mise en scène prend forme peu à peu dans le cadre d'ateliers avec les comédiens. Elle laisse toute la place aux hésitations, quitte à les retirer par la suite. Moi qui ai tendance à raturer les mots mentalement avant même de les écrire, je laisse maintenant plus d'espace aux différentes possibilités du texte avant de prendre des décisions. Mais Brigitte m’a aussi permis de voir que mon écriture est d'une grande violence, même si je ne pratique pas une écriture de l'excès, du débordement, si je peux employer cette expression. Mon écriture est concise, je pèse tous les mots; c'est l'influence de Flaubert, bien sûr. Mais on peut être violent sans crier, sans hurler. Brigitte a également attiré mon attention sur les moments d'humour dans mon écriture, ce qui est rarement mis en évidence.

voIX ET IMAGES Le commentaire de Brigitte Haentjens au sujet de la violence dans votre œuvre est intéressant, mais en même temps il me laisse un peu perplexe. Il me semble que c'est plutôt la blessure, l'abandon et la mort qui hantent le côté sombre de votre œuvre. Comment se manifeste cette "grande violence» dans votre œuvre? LOUISE DUPRÉ Je suis d'accord avec vous pour affirmer que mon écriture se fonde d'abord et avant tout sur la blessure, l'abandon et la mort. Mes deux romans le montrent, de même que certains recueils de poésie, surtout Noir déjā, Tout près et Une écharde sous ton ongle. Mais il y a aussi une violence dans ce que j'écris : dans La memoria et dans La Voie lactée, deux personnages se suicident violemment. Et il y a une grande violence dans l'attitude de Noëlle, la jeune sœur d'Emma, qui s'enfuit sans donner de nouvelles à sa famille par la suite, une violence aussi dans l'attitude de Jérôme qui, un soir, apprend à Emma qu'il part et ne reviendra plus. De la même façon, dans La Voie lactée, l'abandon du père est violent, puisqu'il oblige Anne à tenir sa place auprès de la mère. Anne se séparera d'elle, ce qui est nécessaire, mais cette séparation ne peut que témoigner d'une certaine violence. Dans Noir déjà, la suite poétique sur la ville montre la violence urbaine. Mais c'est bien sûr Tout comme elle qui remporte la palme de la violence, une violence qui passe dans de petites phrases, de petits gestes entre mère et fille. Le titre de travail de ce projet était d'ailleurs Ravage, mais c'était un titre qui n’aurait pas été compris...

J'ai eu l'occasion de revoir Tout comme elle récemment, lors du lancement de la vidéo du spectacle, réalisée par Jean-Pierre Masse. Peut-être la violence est-elle plus explicite visuellement que quand on lit le texte. Chose sûre, certains tableaux portés par une voix de mère, comme d'autres portés par une voix de fille, sont très durs. Pour le deuxième acte, je me suis d'ailleurs inspirée de la relation de madame Claudel avec ses deux filles, Camille et Louise. Relation dévastatrice pour Camille, on le sait. 
Votre question me fait réfléchir aussi à la violence dans la société. On est habitués à considérer comme violents des faits spectaculaires: des assassinats, du terrorisme, des viols, etc. Mais il se vit aussi beaucoup de violence dans les rapports humains: les rapports amoureux, les rapports entre parents et enfants, et même les relations en milieu de travail - une remarque méchante, blessante, par exemple. La violence et la blessure sont étroitement liées. Ce qui m'intéresse dans l'écriture, c'est la violence ordinaire, celle qu'on ne remarque pas parce qu'elle fait partie de la vie, qu'elle ne porte pas le nom de violence. Ceci dit, je ne vise pas à montrer la violence pour la violence : je trouve qu'actuellement, il y a dans notre culture une valorisation de l'esthétique de la violence parce que celle-ci est rentable. On aime les films "coup-de-poing», les œuvres "bruyantes", on confond souvent la force de l'imaginaire avec les situations extrêmes. Je ne veux surtout pas en rajouter, ni exposer la violence pour l'exposer, mais essayer de la comprendre. J'essaie de mettre une tension dans mes textes entre cette présence de la violence - et parfois de la cruauté - et la douceur, un mot d'un autre siècle mais qui, pour moi, est important et qu'il faut rapatrier.

voIX ET IMAGES Rapatrier la douceur... Il est vrai que le mot n'est pas à la mode, qu'on en parle peu. Pourquoi cette notion est-elle importante pour vous?

LOUISE DUPRÉ Il me semble qu'il faut réhabiliter la douceur, qui va de pair avec la convivialité, l'attention aux autres et au monde - à la nature, en particulier. Cela convoque l'usage qu'on fait du temps: prendre son temps, redonner place à la dolce vita. Il y a quelques décennies, on avait prédit une société des loisirs, mais on en est loin: le monde tourne de plus en plus vite, les gens travaillent de plus en plus... L'écriture est un art de la lenteur. Et ma vision de la douceur a nécessairement à voir avec le souci que j'ai de donner un espace, dans mes textes, aux petites choses, aux détails. Prendre le temps de vivre, c'est d'abord prendre le temps de regarder autour de soi, échapper à l'enfermement, ce qui nous permet aussi d'échapper à ce que Paul Chamberland appelle l'autisme social.

voix ET IMAGES Revenons à la pièce Tout comme elle. Comment s'explique, selon vous, son grand succès?

LOUISE DUPRÉ Le succès est dû à la mise en scène, très inventive, très ludique, d'une grande beauté. Brigitte Haentjens en a fait une véritable chorégraphie en regroupant cinquante comédiennes très connues, qui se sont données au spectacle avec une extrême générosité. Le texte se présente en tableaux et Brigitte a représenté, de façon picturale souvent, ces tableaux en mouvement. Les comédiennes ont dit qu'il s'agissait d'une des performances les plus difficiles qui leur aient été demandées. Même si elles n'avaient pas de longs textes à apprendre, elles restaient sur scène durant toute la durée du spectacle. Elles ne pouvaient être déconcentrées.

voix ET IMAGES Tout comme elle a mérité le prix de l'Association québécoise des critiques de théâtre. C'est donc une pièce qui a attiré autant le grand public que les spécialistes de théâtre. Doit-on s'étonner de la diversité du public qui a vu et apprécié la pièce?

LOUISE DUPRÉ Les deux premières semaines, c'est un public habitué au thêâtre qui est venu voir le spectacle. Puis peu à peu, un autre public l'a remplacé: ce qu'on appelle habituellement le grand public. Des femmes qui venaient avec leurs filles ou 
avec leur mère, parfois trois générations étaient présentes dans la salle. Parfois, les femmes avaient invité leur conjoint, leurs frères, leurs amis. Certains, certaines n'avaient jamais mis les pieds dans une salle de théâtre, ce qui m'émouvait beaucoup, moi qui crois à l'engagement de la littérature. Mais c'est la preuve même que les gens peuvent comprendre et apprécier des textes denses, des mises en scène avant-gardistes, n'en déplaise à ceux qui gèrent l'industrie culturelle et qui ont l'impression qu'il faut niveler par le bas.

voIX ET IMAGES Vous commentez avec enthousiasme le fait qu'un public varié a vu la pièce. Doit-on en conclure que vous écrivez pour atteindre de nombreux lecteurs? LOUISE DUPRÉ Mes premiers livres ont été des recueils de poésie. Les poètes savent que leur lectorat sera restreint. En ce sens, je n'ai jamais voulu écrire pour le grand nombre. J'écris parce que c'est pour moi une nécessité, que j'en ressens le besoin pour mon équilibre, peu importe le nombre de personnes qui me liront. C'est pour cette raison que j'ai voulu avoir un travail rémunérateur: pour préserver dans l'écriture mon espace de liberté, pour n'être pas obligée de m'adonner à une écriture alimentaire. Ne pas être obligée non plus de répondre à la demande, par exemple publier rapidement parce qu'il le faut pour être subventionné. Les écrivains qui veulent vivre de leur plume connaissent des conditions difficiles. Des études montrent clairement que beaucoup sont pauvres. J'ai une grande admiration pour eux...

Cela dit, je n'ai jamais voulu tomber dans l'autre travers, c'est-à-dire m'en tenir aux happy few. Il peut y avoir dans cette attitude un snobisme certain. On est trop profond, trop hermétique, trop avant-gardiste pour être compris. On est audessus de la mêlée. Ma position reste d'écrire les livres qui me viennent sans avoir un "plan de carrière", ce mot tellement à la mode aujourd'hui, même dans le domaine des arts. En espérant tout de même rejoindre des lecteurs, ceux et celles qui voudront bien accueillir ma parole.

voıx ET IMAGES Effectivement, vous n'hésitez pas à dévier de votre parcours habituel : vous avez collaboré non seulement avec Brigitte Haentjens, mais aussi avec des artistes visuels, des cinéastes, vous avez publié des livres d'artiste, etc.

LOUISE DUPRÉ Oui, il est important pour moi de collaborer avec des artistes d'autres disciplines, parce que leur façon de voir me permet d'interroger ma pratique, d'innover. Présentement, je travaille avec Mario Côté, un artiste qui est professeur à l'École des arts visuels et médiatiques à l'UQAM. Nous avons des échanges passionnants: ses réflexions sont très stimulantes, particulièrement en ce qui concerne les liens entre l'écriture et les arts médiatiques, et j'espère que mes réflexions l'alimentent aussi.

Mais j'ai également collaboré avec des poètes. J'ai écrit le recueil de poésie "Quand on a une langue on peut aller à Rome» avec Normand de Bellefeuille et le texte du livre d'artiste Parfois les astres avec Denise Desautels. Nous avons procédé différemment dans les deux cas mais, lors de ces deux expériences, l'écriture à quatre mains a été un réel bonheur. Dans Parfois les astres, j'intervenais dans les poèmes de Denise, et elle de même. Le résultat est étonnant: ce ne sont ni des poèmes de Denise Desautels ni des poèmes de Louise Dupré, mais des poèmes de l'auteure $X$, qui est née de notre processus même. Pour une écrivaine - et une professeure de création -, il s'agit d'une expérience qui donne à réfléchir. 
VoIX ET IMAGES Vous enseignez des cours de création à l'UQAM. Est-ce qu'il y a un lien entre votre pédagogie et votre pratique d'écriture?

LOUISE DUPRÉ La vision de la création que j'aborde a nécessairement un lien avec ma pratique d'écriture : comment pourrait-il en être autrement? Pour ma part, la place de la subjectivité est centrale dans mon enseignement. J'essaie de favoriser chez mes étudiants la remise en question des idées reçues, d'un conditionnement familial et social, de leurs certitudes personnelles. Mais je tente aussi de leur faire prendre conscience de leur vision esthétique. En fait, s'approcher d'une écriture personnelle passe nécessairement par une entreprise de déconstruction. On parle habituellement d'ateliers d'écriture : ma métaphore à moi serait plutôt celle du chantier, comme pour Annie Dillard. On démolit et on reconstruit, pierre à pierre. Quand j'étais enfant, je passais des heures et des heures à dessiner des maisons. L'architecture m'a toujours vivement intéressée...

VoIX ET IMAGES Est-ce pour cette raison qu'Anne Martin, dans La Voie lactée, est architecte?

LOUISE DUPRÉ Sans doute... Je n'y ai pas réfléchi consciemment quand j'ai écrit $L a$ Voie lactée, mais il y a chez elle, comme chez moi, un désir de rigueur toute mathématique et un amour de la création. Il y a aussi chez Anne Martin, du moins je crois, un désir de créer des lieux habitables. Pour elle, ce sont des lieux physiques; pour moi, des espaces symboliques. Le titre de travail de La memoria était d'ailleurs «La demeure». J'ai signé aussi des recueils de poésie où les titres étaient Où, Chambres, Tout près. Même La Voie lactée représente un lieu...

VoIX ET IMAGES Votre écriture est marquée par des oppositions fondamentales. D'une part, le suicide et la mort sont des thèmes récurrents mais, d'autre part, l'espoir, la résilience, le renouvellement sont toujours présents au sein même du deuil et de la perte. Est-ce que cette antithèse traduit votre vision du monde? Ou peut-être votre vision de la vie intérieure?

LOUISE DUPRÉ En fait, mon écriture est une écriture funambule, qui avance sur un fil, en essayant de garder l'équilibre. Ce que je tente aussi dans ma vie, inutile de me le cacher. Et le temps faisant son œuvre, j'y arrive de mieux en mieux. Mais dans l'écriture comme dans la vie, il n'y a pas de certitudes : l'espoir, seulement, de ne pas perdre pied... Il y a du noir dans mes textes mais, en contrepartie, l'amour est très présent, sous toutes ses formes: amour pour un homme, pour un enfant, amitié pour un homme ou une femme, etc. Ce sont des figures de la présence, de l'espoir, d'une force vitale...

VoIX ET IMAGES Un autre thème au centre de la poétique de votre œuvre est celui du corps, particulièrement le corps vieillissant. Comment expliquez-vous l'importance que vous accordez au corps?

LOUISE DUPRÉ Je ne suis pas une personne cérébrale, même si je me considère comme une «intellectuelle». La perception sensuelle, le pulsionnel occupent une grande place dans ma compréhension de la réalité. D'abord, j'éprouve une sensation, puis une émotion, qui est ensuite décantée, si je puis dire, mise à distance, par la raison. Cet état de fait se sent dans mon écriture, où les sens sont très présents : la vue, mais aussi l'ouïe, le goût, l'odorat. Et cela se fait sentir aussi dans le travail du style: par le travail du rythme, particulièrement, par la musique de la langue. Dans ces 
conditions, le thème du corps ne peut que m'intéresser vivement. Mon premier recueil s'intitulait La peau familière. Dans Une écharde sous ton ongle, j'ai travaillé sur le vieillissement. Parce que ce fait me concerne directement - je vieillis, personne n'y échappe -, mais aussi parce que j'ai perdu ces dernières années plusieurs amis, ce qui m’a nécessairement renvoyée à ma propre mortalité... Plus on vieillit, plus on se rend compte qu'on ne peut échapper au corporel. C'est le corps qui a le mot de la fin.

voIX ET IMAGES Dans la nouvelle «Pas à pas» (L'été funambule), on lit: «Vous dites souvent qu'il faut arriver à connaître les possibles de son écriture.» Comment concevez-vous les possibles de votre écriture?

LOUISE DUPRÉ J'ai souvent dit à mes étudiants qu'on n'écrit pas ce qu'on veut, mais ce qu'on peut. Et connaître les possibles de son écriture vient petit à petit: c'est le travail d'une vie. Il y a des œuvres que j'admire énormément, mais qui sont aux antipodes de ce que je pourrais écrire, les œuvres plus cérébrales, par exemple, les sagas historiques, tout aussi bien, la poésie formaliste, même si la forme est pour moi primordiale dans l'écriture. Ce qui m'intéresse, c'est vraiment l'exploration de la subjectivité dans ses méandres, dans ses inquiétudes, dans ses espoirs, dans ses déceptions. Non pas une écriture de type psychologique, mais plutôt une écriture du fantasme, ouverte à l'inconscient. Quand j'ai écrit Tout comme elle, j'ai lu beaucoup d'ouvrages psychanalytiques et sociologiques sur la relation mère-fille. Mais je suis consciente qu'on ne connaît jamais tous les possibles de l'écriture. En ce sens, la pratique de différents genres littéraires m'aide à continuer mon parcours. Récemment, par exemple, j'ai commencé à aborder des sujets plus près du politique dans mes nouvelles: cette thématique est apparue sans doute parce que le monde dans lequel on vit est de plus en plus menacé. Mais j'ai toujours été intéressée par la question politique. J'essaie de rester ouverte au monde. Un écrivain ne peut être coupé du monde.

voIX ET IMAGES Se pencher sur le politique - dans sa dimension sociale - , n'est-ce pas aller à l'encontre de la nature intimiste de votre écriture?

LOUISE DUPRÉ Entendons-nous bien: je n'ai pas le projet d'écrire une politiquefiction (rires). Et, de toute façon, la question du politique a toujours traversé ma conception de l'écriture. Le précepte féministe des années 1970, "Le privé est politique», rend bien compte de ma vision. Il ne faut pas confondre le privé et l'intime, bien sûr, mais il n'en reste pas moins que les deux notions ne sont pas antithétiques. La société dans laquelle nous vivons a nécessairement des résonances sur nous, sur la façon dont nous réagissons, sur nos convictions profondes. Actuellement, je trouve que la société néo-libérale exerce sans en avoir l'air une grande violence sur l'individu, ce dont je rends compte dans certains de mes textes. Nécessité de l'excellence constante, sinon de la perfection, travail effréné, encouragement à la consommation, jusque dans le corps, qui doit être refait périodiquement, jusque dans la sexualité, qui fonctionne au rythme de la performance: cette situation entraîne beaucoup de problèmes, dont des problèmes psychiques accrus chez les gens, particulièrement chez les jeunes. Je vis de plus en plus en marge des valeurs de la société actuelle, et j'en éprouve de plus en plus de douleur, je dois le dire.

Je ne veux surtout pas devenir moraliste, mais je ne peux être aveugle non plus. En ce sens, le travail littéraire que je veux faire en est un de résistance aux 
valeurs de la société actuelle, résistance qui prend plusieurs aspects: soit le fait de dire l'inquiétude, comme dans la poésie; soit le fait de peindre des personnages qui sont aux prises avec certaines difficultés; soit par l'écriture de l'essai - je prépare actuellement un essai, ou ce qu'on aurait appelé dans les années 1970 une théoriefiction, sur la douleur. Lors d'un festival de poésie à Berlin, en juin 2007, j'ai vu une magnifique exposition sur la douleur, qui conviait à la fois les arts visuels et la médecine. J'ai connu un chemin de Damas. Je me suis demandé si, à Montréal, où règne actuellement le stand-up comic, on aurait osé annoncer, à tous les coins de rue, «Douleur, douleur, douleur »... Ma réponse a été évidemment non. Pour regarder la douleur en face, il faut être capable aussi de regarder la société dans laquelle on vit. Et accepter de se regarder...

voIX ET IMAGES Ceux qui vous connaissent verront certains éléments autobiographiques dans votre œuvre (les allusions par exemple à votre fille et à votre enfance). Pratiquez-vous consciemment une écriture autobiographique ou bien s'agit-il de bribes de réel qui s'inscrivent dans la fiction et la poésie?

LOUISE DUPRÉ Un étudiant d'un collège m'a écrit il y a plusieurs années pour me demander si La memoria était autobiographique. Je lui ai répondu: "Oui et non.» Déçu, il m’a répliqué que, dans sa dissertation, il lui fallait répondre "Oui» ou «Non». Je lui ai dit alors que le roman n'était pas autobiographique. Si je me permets de rappeler cette anecdote, c'est qu'elle me semble illustrer toute la complexité de la question. Toute œuvre est, d'une certaine façon, autobiographique. Mais c'est une question de degrés. C'est aussi une question qui a à voir avec les genres littéraires. Dans la poésie, par exemple, les études sur le sujet lyrique nous montrent que le je poétique a des liens directs avec le biographique, mais le met à distance, le transcende, pour rejoindre l'universel... Dans ma prose, je m'ennuierais, il me semble, si je devais m'en tenir aux faits de ma vie. Et d'ailleurs, la mémoire ne déforme-t-elle pas toujours les faits? Ne recrée-t-elle pas, jusqu'à un certain point, le passé? Ainsi, je ne nie aucunement qu'il y ait une base autobiographique, mais je ne m'en tiens pas à cela. Plusieurs de mes nouvelles, par exemple, viennent de ce qu'on m'a raconté. Ce qu'on entend, ce qu'on lit fait aussi partie de soi... De même que la musique qu'on écoute, que les pièces de thêâtre qu'on voit. Je dirais que mon écriture vient de mon expérience des choses plus que de l'autobiographie. L'important, dans une entreprise d'écriture, c'est de maintenir la bonne distance avec la réalité, celle qui nous permet d'écrire un texte qui se tient.

Cela dit, je suis une personne pudique. Cela vient de mon éducation. Je ne me livre pas facilement. Et j'éprouve le besoin de garder une certaine distance avec les faits plus autobiographiques dans mon travail pour protéger les personnes qui m'entourent. Pour me protéger aussi. Je crois à l'authenticité d'un texte, mais je n'éprouve pas le besoin de dire la vérité, toute la vérité. La fiction est d'ailleurs souvent la meilleure façon d'atteindre à la vérité subjective.

voIX ET IMAGES En lisant, dans ce dossier, votre inédit "La porte fermée», je crois déceler un changement dans votre démarche. D'une écriture intimiste, vous passez à un questionnement sur la relation entre l'histoire et l'art. Vous abordez également le grand thème de la mémoire (ou de l'amnésie) collective. Est-ce une déviation temporaire ou bien une nouvelle direction? Quels sont en fait vos projets d'écriture? 
LOUISE DUPRÉ Depuis le début de mon parcours, ce sont habituellement les mêmes thèmes qui m'intéressent. Seulement, selon les genres littéraires, les livres, les périodes de ma vie, certains prennent le premier plan dans mon écriture, alors que d'autres restent à l'arrière-plan. Le recueil La peau familière commençait par une suite poétique où une femme, qui regardait le journal télévisé avec sa fille, voyait des images du massacre de Sabra et Chatila. Dans La memoria, madame Girard, passionnée par l'histoire, va jusqu'en Crète pour retrouver sa sérénité après le suicide de son mari. Dans La Voie lactée, Alessandro Moretti a passé sa vie à fouiller les ruines de Carthage, pour exhumer l'histoire des Phéniciens, un peuple de voyageurs qui a sillonné les mers, mais dont on ne sait malheureusement plus grand-chose aujourd'hui. La situation du Québec dans l'avenir pourrait bien se comparer à celle des Phéniciens si on n'assure pas une mémoire culturelle collective solide.

Mémoire, oubli: deux postures aussi bien face à la petite histoire qu'à la grande. Je n'ai jamais tenté de séparer l'intime du collectif, j'ai plutôt essayé de les mettre en relation. Pourtant, il est vrai que, dans "La porte fermée», la dimension historique passe au premier plan, et j'irai peut-être de plus en plus dans ce sens-là. Cette nouvelle est paradoxalement l'un des textes les plus autobiographiques qu'il m'ait été donné d'écrire. L'an dernier, lors de mon voyage en Allemagne, j'ai visité Munich et je me suis rendue à Dachau. Ce séjour a exacerbé mon rapport à la douleur. À côté de tout ce que je connaissais de l'Allemagne hitlérienne, j'ai découvert des faits que j'ignorais: par exemple, l'histoire de ces étudiants munichois - de l'âge de nos étudiants - qui militaient contre le régime nazi, qui ont été pris et ont trouvé la mort après des tortures atroces. Aucun peuple n'est à l'abri de l'horreur. Dans nos sociétés actuelles, il faut garder les yeux grands ouverts.

Mais je m'éloigne de l'écriture... Ou peut-être pas tout à fait, puisqu'il est vrai que l'écriture vient pour moi d'une nécessité de raviver la mémoire, sa propre mémoire, toujours liée à la mémoire collective. Je voudrais continuer à travailler dans ce sens. Je prépare actuellement un roman sur la maladie mentale, je lis beaucoup: des romans, des journaux intimes, des faits sur les traitements imposés au cours des années au Québec, etc. Durant les années 1970, on croyait que, si les individus réalisaient leurs désirs, il y aurait moins de maladie mentale. C'était très naïf. Au contraire, malgré les progrès de la psychanalyse et de la médecine, il me semble qu'il $\mathrm{y}$ a de plus en plus de gens qui souffrent de problèmes mentaux, de tous les âges, de toutes les classes sociales. Je voudrais aborder cette question-là. Mais il s'agit d'un projet parmi d'autres : j'ai tant de livres à écrire encore. J'espère vivre très vieille... 